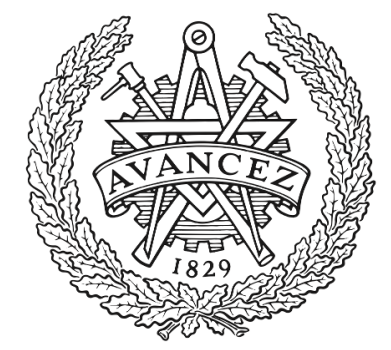

\title{
CHALMERS
}

UNIVERSITY OF TECHNOLOGY

\section{Influence of thermal expansion on potential and rotational components of turbulent velocity field within and upstream of premixed flame brush}

Downloaded from: https://research.chalmers.se, 2023-04-26 09:46 UTC

Citation for the original published paper (version of record):

Lipatnikov, A., Sabelnikov, V., Nikolay, N. et al (2019). Influence of thermal expansion on potential and rotational components of turbulent velocity

field within and upstream of premixed flame brush. Proceedings of the Eleventh Mediterranean

Combustion Symposium, MCS11: 1-12

N.B. When citing this work, cite the original published paper. 


\title{
INFLUENCE OF THERMAL EXPANSION \\ ON POTENTIAL AND ROTATIONAL COMPONENTS OF TURBULENT VELOCITY FIELD WITHIN AND UPSTREAM OF PREMIXED FLAME BRUSH
}

\author{
A.N. Lipatnikov*, V.A. Sabelnikov**, N. Nikitin***, S. Nishiki**** and T. \\ Hasegawa $* * * * *$ \\ vladimir.sabelnikov@onera.fr \\ *Chalmers University of Technology, Gothenburg, 41296, Sweden \\ **ONERA - The French Aerospace Lab., F-91761 Palaiseau, France \\ ***Moscow State University, Moscow, Russian Federation \\ ****Kagoshima University, Kagoshima 890-0065, Japan \\ *****Nagoya University, Nagoya 464-8603, Japan
}

\begin{abstract}
Direct Numerical Simulation (DNS) data obtained earlier from two statistically stationary, 1D, planar, weakly turbulent premixed flames are analyzed in order to examine the influence of combustion-induced thermal expansion on the flow structure within the mean flame brushes and upstream of them. The two flames are associated with the flamelet combustion regime and are characterized by significantly different density ratios, i.e. $\sigma=7.53$ and 2.5. The Helmholtz-Hodge decomposition is applied to the DNS data in order to extract rotational and potential velocity fields. Comparison of the two fields shows that combustion-induced thermal expansion can significantly change the local structure of the incoming constantdensity turbulent flow of unburned reactants by significantly increasing the relative magnitude of the potential velocity fluctuations when compared to the rotational velocity fluctuations in the flow. Such effects are documented not only within the mean flame brush, but also well upstream of it. The effect magnitude is increased by the density ratio $\sigma$, with the effects being well (weakly) pronounced at $\sigma=7.53$ (2.50, respectively). Moreover, the potential and rotational velocity fields can cause opposite variations of the local area of an iso-scalar surface $c(\mathbf{x}, t)=$ const within flamelets by generating the local strain rates of opposite signs.
\end{abstract}

\section{Introduction}

Almost seven decades ago, the seminal work by Karlovitz et al. [1] and by Scurlock and Grover [2] initiated research into the influence of premixed combustion on turbulent flow and eventual increase in burning rate due to the so-called flame-generated turbulence. Progress obtained in this area is reviewed elsewhere [3-6], but, over decades, the focus of the vast majority of studies of the flame-generated turbulence was placed on the flow downstream of the instantaneous (or local) flame within the mean flame brush.

About a decade ago, Swaminathan and Grout [7] found that, due to dilatation caused by local thermal expansion, scalar gradients within the local flame could preferentially be aligned with the direction of the most expansive strain rate, whereas scalar gradients align preferentially with the most compressive strain rate in a constant-density turbulent flow. Subsequently, the alignment issue was addressed in a series of Direct Numerical Simulation (DNS) and experimental studies, which confirmed the aforementioned finding, e.g. see Refs. [6,8-11] and references cited therein. However, available data indicate that such effects are restricted to local flames, but are not observed in unburned gas upstream of a flame [9-11]. 
As far as eventual increase in burning rate due to the influence of combustion on turbulence is concerned, it is worth stressing that a flame propagates into unburned gas. Thus, perturbations of the velocity field in the unburned gas upstream of the flame are required in order for such a phenomenon to arise. However, little is yet known about the influence of combustion on the constant-density turbulent flow of unburned gas upstream of a premixed flame.

Recently, some of the present authors [12,13] analyzed the Nagoya DNS database [14,15] and showed that acceleration of the unburned gas by combustion-induced pressure gradient yielded unburned mixture fingers that deeply intruded into the products, thus, significantly increasing flame surface area, turbulent burning rate, and mean flame brush thickness. Appearance of such unburned mixture fingers was also documented in subsequent experiments [16,17]. However, this phenomenon is mainly controlled by the influence of combustion-induced pressure perturbations on the mean velocity conditioned to the unburned reactants, with the governing physical mechanism being basically similar to the physical mechanism of the hydrodynamic instability [18-20] of laminar premixed flames.

Analysis of the same DNS data $[14,15]$ has also shown that (i) the second-order structure functions of the velocity field, conditioned to the unburned reactants, are substantially affected by combustion-induced thermal expansion [21] and (ii) the $Q$-criterion is predominantly negative in the unburned reactants in the vicinity of local flames if the density ratio $\sigma=\rho_{u} / \rho_{b}=7.53$, but such effects are rarely pronounced if $\sigma=2.5$ [6]. Here, $Q=$ $\omega^{2} / 4-S^{2} / 2$ [22], $\omega^{2}=\boldsymbol{\omega} \cdot \boldsymbol{\omega}$ and $\boldsymbol{\omega}=\nabla \times \mathbf{u}$ are enstrophy and vorticity, respectively, $S^{2}=S_{i j} S_{i j}$ is the total strain, $S_{i j}=\left(\partial u_{i} / \partial x_{j}+\partial u_{j} / \partial x_{i}\right) / 2$ is the rate-of-strain tensor, the summation convention applies for repeated indexes, $\mathbf{u}$ is the flow velocity vector, and subscripts $u$ and $b$ designate unburned reactants and equilibrium combustion products, respectively.

The aforementioned trend for $Q$ to become negative in the vicinity of local flames indicates that combustion-induced thermal expansion makes total strain larger than enstrophy and, therefore, changes the local structure of the incoming turbulent flow of unburned reactants. Such changes could be attributed to pressure perturbations, which appear due to density drop in the local flame and affect the flow field upstream of the flame. Indeed, since the pressure gradient $\nabla p$ (i) directly affects the velocity field, i.e., the Navier-Stokes equations involve $\nabla p$, but (ii) does not directly affect the vorticity field if the local density $\rho=\rho_{u}$ is constant, i.e., the transport equation for vorticity [5] does not involve $\nabla p$ in such a case (while the pressure perturbations can indirectly affect the vorticity field by changing vortex stretching term in the transport equation for $\boldsymbol{\omega})$; the velocity perturbations upstream of the flame might be expected to be predominantly potential (irrotational). However, to the best of the present authors' knowledge, nobody has yet cogently proved that combustion-induced thermal expansion perturbs the local structure of the incoming turbulent flow of unburned reactants by increasing the relative magnitude of potential velocity fluctuations therein. The present paper aims at filling this gap by further analyzing the Nagoya DNS data $[14,15]$ and applying the Helmholtz-Hodge decomposition to computed velocity fields $\mathbf{u}(\mathbf{x}, t)$.

The choice of this DNS database, which may appear to be outdated when compared to recent DNS data [23-31] generated in the case of complex combustion chemistry and a high ratio of the rms turbulent velocity $u^{\prime}$ to the laminar flame speed $S_{L}$, requires comments.

Since the focus of the following discussion is placed on the influence of combustioninduced thermal expansion on the velocity, pressure, vorticity, and enstrophy fields upstream of reaction zones, detailed description of complex combustion chemistry within such zones appears to be of secondary importance when compared to two other major requirements. First, in order to make the studied thermal expansion effects as strong as possible, heat release and density drop should be localized to sufficiently thin zones and the velocity jumps across such 
zones should be sufficiently large when compared to the rms turbulent velocity $u^{\prime}$. In other words, the flamelet regime [32-34] of premixed turbulent combustion associated with a low $u^{\prime} / S_{L}=0(1)$ should be addressed. The selected DNS data are indeed associated with this regime, as discussed in details elsewhere [35], whereas the vast majority of recent very advanced DNS studies attacked other combustion regimes characterized by a large $u^{\prime} / S_{L}$. Second, to better explore the thermal expansion effects, data obtained at significantly different density ratios $\sigma$ are required. The selected DNS database does satisfy this requirement, because cases of $\sigma=2.5$ and 7.53 were simulated, with all other things being roughly equal. Therefore, the selected DNS data appear to be fully adequate to the major goal of the present work.

The paper is organized as follows. In the next section, the DNS attributes are summarized. A method used to decompose the velocity field into rotational and potential components is reported in Section 3. Results are discussed in Section 4, followed by conclusions.

\section{Direct Numerical Simulations}

Since the DNS data were discussed in details elsewhere [14,15] and were already used by various research groups [12,13,21,35-50], let us restrict ourselves to a very brief summary of those compressible 3D simulations. They deal with statistically 1D and planar, equidiffusive, adiabatic flames modeled by unsteady continuity, Navier-Stokes, and energy equations, supplemented with the ideal gas state equation and a transport equation for the mass fraction $Y$ of a deficient reactant. Temperature-dependence of molecular transport coefficients is taken into account, e.g., the kinematic viscosity $v=v_{u}\left(T / T_{u}\right)^{0.7}$, where $T$ is the temperature. The Lewis Le and Prandtl $\operatorname{Pr}$ numbers are equal to 1.0 and 0.7 , respectively. Combustion chemistry is reduced to a single reaction. Therefore, the mixture state is characterized with a single combustion progress variable $c=\left(T-T_{u}\right) /\left(T_{b}-T_{u}\right)=1-Y / Y_{u}$.

The computational domain is a rectangular box $\Lambda_{x} \times \Lambda_{y} \times \Lambda_{z}$, where $\Lambda_{x}=8 \mathrm{~mm}$ and $\Lambda_{y}=\Lambda_{z}=4 \mathrm{~mm}$. It is resolved using a uniform rectangular $(2 \Delta x=\Delta y=\Delta z)$ mesh of $512 \times 128 \times 128$ points. The flow is periodic in $y$ and $z$ directions. The flames propagate from right to left.

Using an energy spectrum $E(\kappa)$ proposed by Kraichnan [51], homogeneous isotropic turbulence is generated [14] in a separate box and is injected into the computational domain through the left (unburned) boundary $x=0$. The generated turbulence is characterized [14] by $u^{\prime}=0.53 \mathrm{~m} / \mathrm{s}$ and an integral length scale $L=3.45 \mathrm{~mm}$. Accordingly, $R e_{t}=u^{\prime} L / v_{u}=$ 96.

At $t=0$, a planar laminar flame is embedded into statistically the same turbulence assigned for the velocity field in the entire computational domain. Subsequently, the mean inflow velocity $U$ is increased twice, $U\left(0 \leq t<t_{1}\right)=S_{L}<U\left(t_{1} \leq t<t_{2}\right)<U\left(t_{2} \leq t\right)$, with $U\left(t_{2} \leq t\right)$ being close to the mean turbulent flame speed $\overline{S_{T}\left(t_{2} \leq t \leq t_{3}\right)}$ in order to keep the flame in the computational domain till the end $t_{3}$ of the simulations.

Three DNS data sets H, M, and L associated with High, Medium, and Low, respectively, density ratios $\sigma$ were originally generated $[14,15]$. Since the focus of the present study is placed on thermal expansion effects, the following discussion will be restricted to results obtained in two cases characterized by the highest and the lowest density ratios, i.e., flame $\mathrm{H}$ $\left(\sigma=7.53, S_{L}=0.6 \mathrm{~m} / \mathrm{s}, \delta_{L}=0.217 \mathrm{~mm}\right)$ and flame $\mathrm{L}\left(\sigma=2.5, S_{L}=0.416 \mathrm{~m} / \mathrm{s}, \delta_{L}=\right.$ $0.158 \mathrm{~mm})$. In both cases, $\overline{S_{T}\left(t_{2} \leq t \leq t_{3}\right)} / S_{L}=1.9$. Here, $\delta_{L}=\left(T_{b}-T_{u}\right) / \max \{|\nabla T|\}$ is the laminar flame thickness. The two flames are well associated with the flamelet combustion regime, e.g., various Bray-Moss-Libby (BML) expressions hold in cases $\mathrm{H}$ and $\mathrm{L}$, see figures 1-4 in Ref. [35]. 
Since turbulence decays along the direction $x$ of the mean flow, the turbulence characteristics are slightly different at the leading edges of the $\mathrm{H}$ and L-flame brushes, e.g., $u^{\prime}=0.33 \mathrm{~m} / \mathrm{s}, \lambda=0.43 \mathrm{~mm}, \eta=0.075 \mathrm{~mm}, D a=17.5$, and $K a=0.06$ in case $\mathrm{H}$ or $u^{\prime}=0.38 \mathrm{~m} / \mathrm{s}, \lambda=0.47 \mathrm{~mm}, \eta=0.084 \mathrm{~mm}, D a=10.0$, and $K a=0.10$ in case L. Here, $D a=\tau_{T} / \tau_{f}$ and $K a=\tau_{f} u^{\prime} / \lambda$ are the Damköhler and Karlovitz numbers, respectively, $\tau_{f}=v_{u} /\left(\operatorname{Pr} S_{L}^{2}\right)$ and $\tau_{T}=\bar{k}^{3 / 2} / \bar{\varepsilon}$ are flame and turbulence time scales, respectively, $\lambda=u^{\prime} / \sqrt{15 v / \bar{\varepsilon}}$ and $\eta=\left(v^{3} / \bar{\varepsilon}\right)^{1 / 4}$ are the Taylor and Kolmogorov length scales, respectively, $k=\left(u_{j} u_{j}-\bar{u}_{j} \bar{u}_{j}\right) / 2$ and $\varepsilon=2 v S_{i j} S_{i j}$ are the turbulent kinetic energy and its dissipation rate, respectively, and the rms turbulent velocity $u^{\prime}$ is equal to $(2 \bar{k} / 3)^{1 / 2}$.

The DNS data are processed as follows. Mean quantities $\bar{q}=\bar{q}(x)$ are averaged over a transverse plane of $x=$ const and over time (221 and 200 snapshots in cases $\mathrm{H}$ and $\mathrm{L}$, respectively, stored during a time interval of $t_{3}-t_{2} \approx 1.5 \mathrm{~L} / u_{0}^{\prime} \approx 10 \mathrm{~ms}$ ). Subsequently, $x$ dependencies are mapped to $\bar{c}$-dependencies using the spatial profiles of the Reynoldsaveraged combustion progress variable $\bar{c}(x)$. Moreover, mean quantities $\langle q\rangle(x, t)$ are averaged over a transverse plane of $x=$ const at the instant $t$.

To explore the influence of combustion-induced thermal expansion on turbulence, the Helmholtz-Hodge decomposition [52-54] is applied to the computed velocity fields $\mathbf{u}(\mathbf{x}, t)$, as described in the next section.

\section{Velocity Decomposition}

If velocity field $\mathbf{u}(\mathbf{x}, t)$ is decomposed into rotational and potential subfields $\mathbf{u}_{r}(\mathbf{x}, t)$ and $\mathbf{u}_{p}(\mathbf{x}, t)$, respectively, then, the following relations should hold

$$
\nabla \times \mathbf{u}_{r}=\nabla \times \mathbf{u}, \quad \mathbf{u}_{p}=\nabla \varphi, \quad \mathbf{u}(\mathbf{x}, t)=\mathbf{u}_{r}(\mathbf{x}, t)+\mathbf{u}_{p}(\mathbf{x}, t) .
$$

However, such a decomposition is not unique, unless the function $\varphi(\mathbf{x}, t)$ is defined.

Accordingly, to solve the problem, the following constraint of orthogonality

$$
\iiint_{V} \mathbf{u}_{r} \cdot \mathbf{u}_{p} d \mathbf{x}=0
$$

of the subfields $\mathbf{u}_{r}(\mathbf{x}, t)$ and $\mathbf{u}_{p}(\mathbf{x}, t)$ is often invoked. This constraint results in the additivity of the kinetic energies of the rotational and potential flow fields, i.e.

$$
\iiint_{V} \mathbf{u} \cdot \mathbf{u} d \mathbf{x}=\iiint_{V} \mathbf{u}_{r} \cdot \mathbf{u}_{r} d \mathbf{x}+\iiint_{V} \mathbf{u}_{p} \cdot \mathbf{u}_{p} d \mathbf{x}
$$

where integration is performed over a domain $V$. Substitution of Eq. (1) into Eq. (2) yields

$$
\iiint_{V} \mathbf{u}_{r} \cdot \nabla \varphi d \mathbf{x}=\iiint_{V} \nabla\left(\varphi \mathbf{u}_{r}\right) d \mathbf{x}-\iiint_{V} \varphi \nabla \cdot \mathbf{u}_{r} d \mathbf{x}=\oiint \oiint_{S} \varphi \mathbf{u}_{r} \cdot \mathbf{n} d S-\iiint_{V} \varphi \nabla \cdot \mathbf{u}_{r} d \mathbf{x}
$$

where $S$ is the boundary of the domain $V$ and the unit vector $\mathbf{n}$ is normal to the boundary. If $\mathbf{u}_{r} \cdot \mathbf{n}=0$ and $\nabla \cdot \mathbf{u}_{r}=0$, then integrals in Eq. (4) vanish, 


$$
\Delta \varphi=\nabla \cdot \mathbf{u}_{p}=\nabla \cdot \mathbf{u}
$$

in the entire domain $V$, and

$$
\left.\frac{\partial \varphi}{\partial n}\right|_{S}=\left.\mathbf{n} \cdot \nabla \varphi\right|_{S}=\left.\mathbf{n} \cdot \mathbf{u}\right|_{S}
$$

on its boundary $S$. The Neumann problem given Eqs. (5) and (6) has a unique solution for $\mathbf{u}_{p}$.

When processing the DNS data, first, the mean axial velocity $\langle u\rangle(x, t)$, which increases due to the density drop in the axial direction $x$, is subtracted from the velocity field $\mathbf{u}(\mathbf{x}, t)$ stored during the simulations. Subsequently, the obtained field $\mathbf{u}^{\prime}(\mathbf{x}, t)$ of velocity fluctuations is decomposed into potential and rotational subfields. For this purpose, the problem given by Eqs. (5) and (6) and applied to the $\mathbf{u}^{\prime}(\mathbf{x}, t)$-field is numerically solved for $\varphi(\mathbf{x}, t)$ at each instant $t$, followed by calculation of the potential $\mathbf{u}_{p}(\mathbf{x}, t)=\nabla \varphi$ and rotational $\mathbf{u}_{r}(\mathbf{x}, t)=\mathbf{u}(\mathbf{x}, t)-\mathbf{u}_{p}(\mathbf{x}, t)$ velocities. At each time step and in each grid points, the validity of Eqs. (1), (3), and $\nabla \cdot \mathbf{u}_{r}=0$ is confirmed with a high precision.

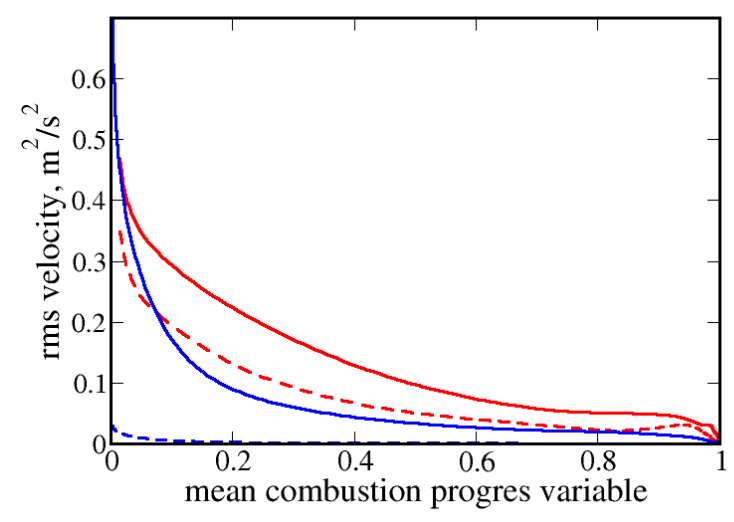

(a)

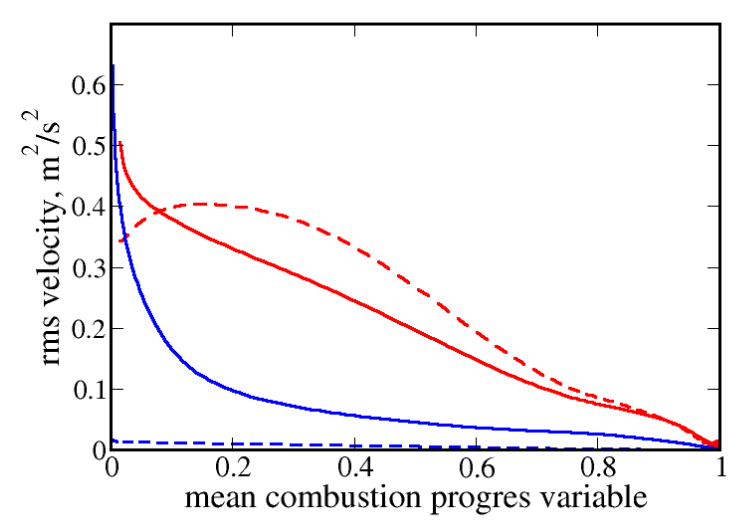

(c)

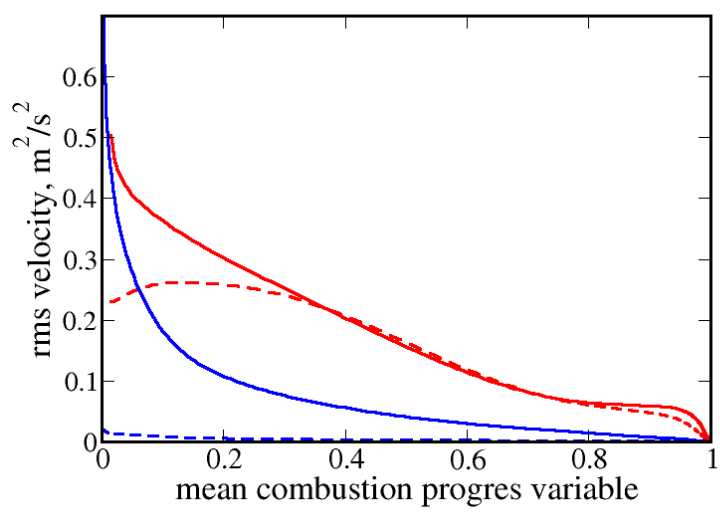

(b)

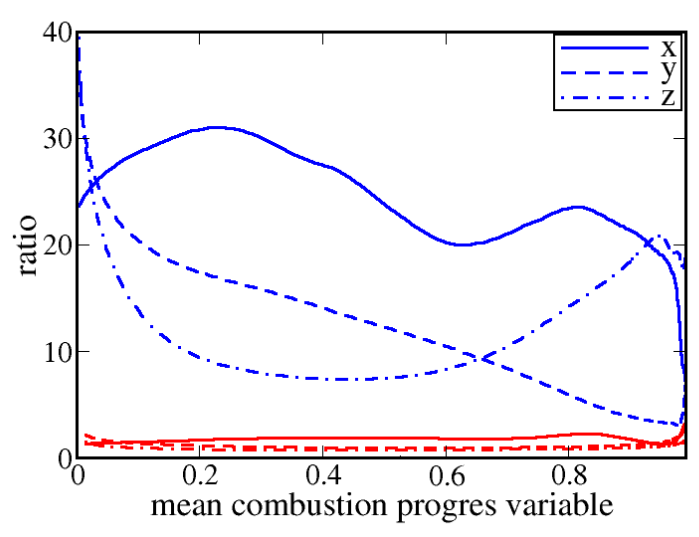

(d)

Figure 1. RMS magnitudes of the potential (dashed lines) and rotational (solid lines) velocities conditioned to unburned gas, i.e. $c(\mathbf{x}, t)<0.03$, within the mean flame brush. Red and blue lines show results computed in cases $\mathrm{H}$ and L, respectively. (a) Axial velocities $u_{p}$ and $u_{r}$, (b) transverse velocities $v_{p}$ and $v_{r}$ in the $y$-direction, (c) transverse velocities $w_{p}$ and 
$w_{r}$ in the $z$-direction, (d) ratios of $u_{r} / u_{p}$ (solid lines), $v_{r} / v_{p}$ (dashed lines), and $w_{r} / w_{p}$ (dotted-dashed lines).

\section{Results and Discussion}

Figures 1a-1c show the rms magnitudes of the rotational (solid lines) and potential (dashed lines) velocities conditioned to unburned gas, i.e. evaluated at $c(\mathbf{x}, t)<0.03$, within the mean flame brush in case $\mathrm{H}$ (red lines) or $\mathrm{L}$ (blue lines). In case $\mathrm{L}$ characterized by a lower $\sigma=2.5$, the rms magnitudes of the rotational velocities are much higher than their potential counterparts similarly to a constant-density turbulent flow. On the contrary, in case $\mathrm{H}$ characterized by a larger $\sigma=7.53$, difference in the rms magnitudes of the rotational and potential axial velocities is much less pronounced, cf. red dashed and solid lines in Fig. 1a. Moreover, the rms magnitudes of the potential transverse velocities can be equal to or even larger than its rotational counterpart within the largest part of the mean flame brush with the exception of its leading edge, see Figs. $1 \mathrm{~b}$ and $1 \mathrm{c}$. These data clearly show that combustioninduced thermal expansion can change the local structure of the incoming turbulent flow of unburned reactants by generating significant potential velocity fluctuations if the density ratio is sufficiently large.

Furthermore, comparison of the rms magnitudes of the rotational velocity fluctuations in cases $\mathrm{H}$ and $\mathrm{L}$, see red and blue, respectively, solid lines in Figs. 1a-1c, indicates that rotational velocity fluctuations conditioned to the constant-density unburned reactants are also significantly increased within the H-flame brush when compared to the L-flame brush. This effect could be attributed to an increase in the vortex-stretching term in the transport equation for vorticity [5] due to the increase in the potential velocity fluctuations.

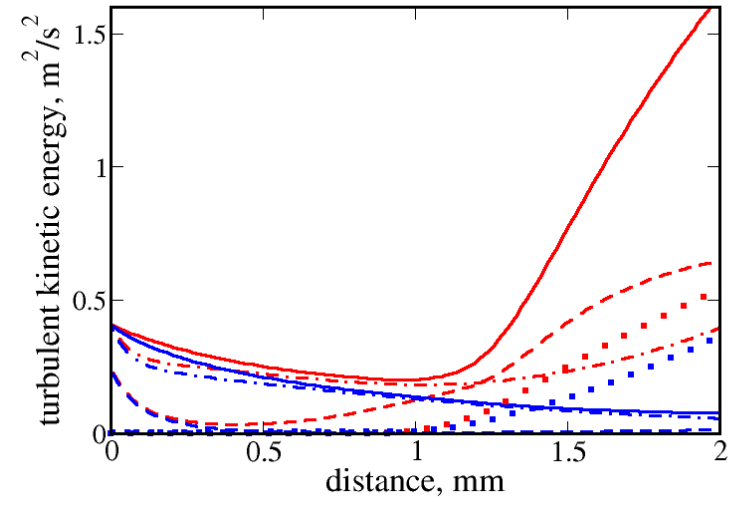

(a)

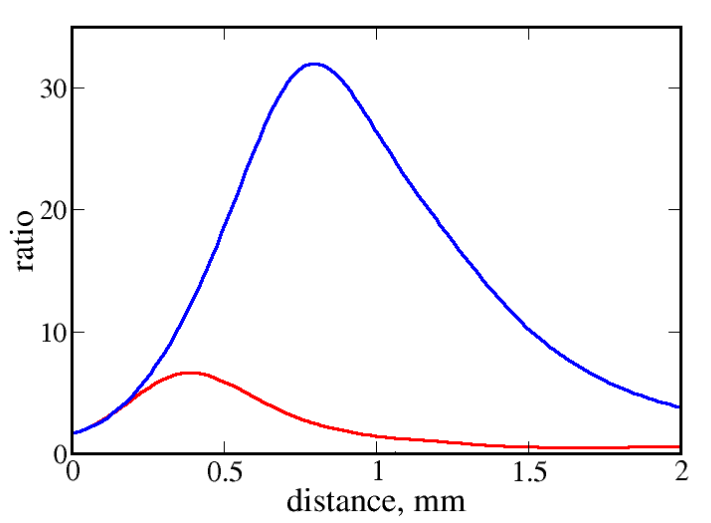

(b)

Figure 2. (a) Spatial profiles of the mean turbulent kinetic energy $\bar{k}(x)$ calculated for the original $\mathbf{u}(\mathbf{x}, t)$, see solid lines, potential $\mathbf{u}_{p}(\mathbf{x}, t)$, see dashed lines, and rotational $\mathbf{u}_{r}(\mathbf{x}, t)$, see dotted-dashed lines, velocity fields conditioned to unburned reactants, i.e. $c(\mathbf{x}, t)<0.03$, as well as spatial profiles of the mean combustion progress variable $\bar{c}(x)$, see dotted lines.

Red and blue lines show results obtained in cases $\mathrm{H}$ and L, respectively.

(b) Ratio of the mean turbulent kinetic energies evaluated for the rotational and potential velocity fields in cases $\mathrm{H}$ (red line) and L (blue line).

Such thermal expansion effects are not restricted to the mean flame brush, but are also observed upstream of it. For instance, Fig. 2a shows that, in the vicinity of the inlet $x=0$ of the computational domain, the rotational turbulent kinetic energy (dotted-dashed lines) is significantly larger than and decays (in the axial direction $x$ ) significantly slower than its potential counterpart (dashed lines). Such trends are typical for non-reacting turbulent flows 
and are very similar in cases $\mathrm{H}$ and $\mathrm{L}$ at $x<0.2 \mathrm{~mm}$. In case $\mathrm{L}$, the same trends are observed even at larger $x<0.8 \mathrm{~mm}$, e.g. see blue solid line in Fig. 2b. Then, a ratio of the rotational and potential turbulent kinetic energies peaks at $x \approx 0.8 \mathrm{~mm}$, see blue solid line in Fig. $2 \mathrm{~b}$, i.e., in the vicinity of the leading edge of the mean flame brush, see the profile of $\bar{c}(x)$ plotted in a blue dotted line in Fig. 2a. At larger $x$, i.e., within the mean flame brush, the ratio decreases probably, due to the thermal expansion effects.

In case $\mathrm{H}$, the thermal expansion effects are much more pronounced within the mean flame brush, cf. red and blue lines in Fig. $2 a$ or $2 b$, and are observed even well upstream of the mean flame brush. Indeed, the ratio of the rotational and potential turbulent kinetic energies peaks at $x<0.4 \mathrm{~mm}$, see red solid line in Fig. $2 \mathrm{~b}$, whereas the leading edge of the mean flame brush is associated with $x \approx 1 \mathrm{~mm}$ in this case, see red dotted line in Fig. 2a. Thus, substantial effects of thermal expansion on the structure of the turbulent flow are observed upstream of the mean flame brush. In particular, significant generation of potential velocity fluctuations is detected at distance of about $0.5 \mathrm{~mm}$ from the leading edge of the mean flame brush in case $\mathrm{H}$.

It is also worth noting that comparison of red and blue dotted-dashed lines in Fig. 2a indicates that the rotational turbulent kinetic energy is slightly larger in case $\mathrm{H}$ than in case $\mathrm{L}$ far upstream of the mean flame brush (already at $x>0.1 \mathrm{~mm}$ ). However, this difference may be controlled by a lower mean inlet velocity $\bar{U}$ of the flow in case $\mathrm{L}$ when compared to case $\mathrm{H}$. It is worth remembering that $\bar{U} \approx \overline{S_{T}\left(t_{2} \leq t \leq t_{3}\right)}$ in both cases, but $\overline{S_{T}\left(t_{2} \leq t \leq t_{3}\right)}=$ 1.15 and $0.79 \mathrm{~m} / \mathrm{s}$ in cases $\mathrm{H}$ and $\mathrm{L}$, respectively. Consequently, turbulence in a fluid volume detected at a distance $x$ from the inlet has decayed during a longer time interval in case $\mathrm{L}$ when compared to case $\mathrm{H}$. Accordingly, the aforementioned difference between the rotational turbulent kinetic energies could be attributed to a longer (shorter) decay of the inlet turbulence in case $\mathrm{L}(\mathrm{H}$, respectively). At $\bar{c} \rightarrow 0$ in Figs. 1a-1c, such differences in the rotational velocity fields are less pronounced, probably, because the $\mathrm{H}$-flame brush is closer to the inlet boundary, cf. red and blue dotted lines in Fig. 2a.

Substantial influence of combustion-induced thermal expansion on the local structure of the turbulent flow within and upstream of a mean flame brush is illustrated in Fig. 3, which shows typical 2D images of the sign of the $Q$-criterion. Figure 3 a indicates that $Q=\omega^{2} / 4-$ $S^{2} / 2$ is predominantly positive far upstream of a flamelet, where the enstrophy $\omega^{2}$ is typically larger than $S^{2}$, but is predominantly negative close to and within the flamelet, thus, implying the local generation of potential velocity perturbations in the incoming turbulent flow of unburned reactants in case $\mathrm{H}$. On the contrary, both positive and negative $Q$ are observed within flamelet in case L, see Fig. 3 b.

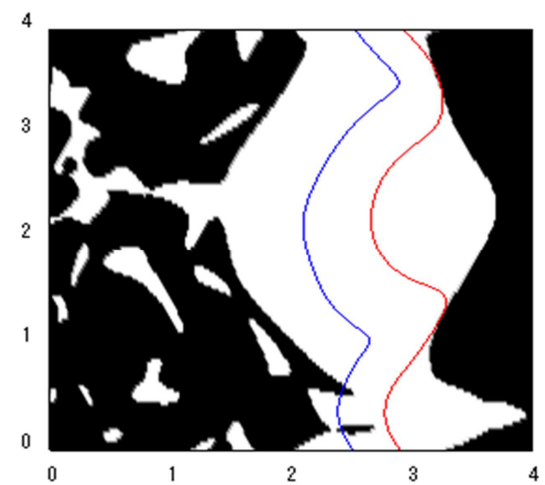

(a)

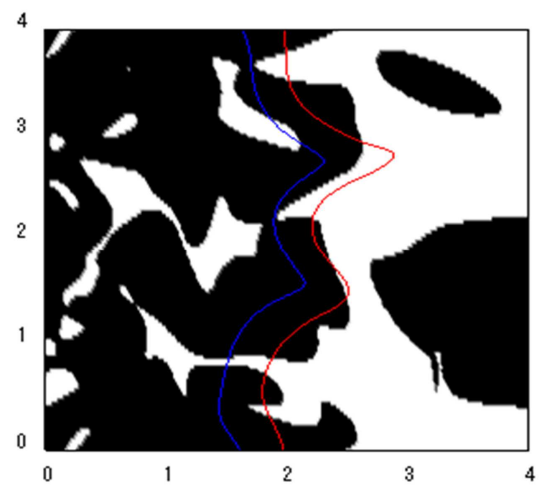

(b)

Figure 3. Typical 2D images of the sign of the $Q$-criterion in cases (a) $\mathrm{H}$ and (b) L. Spatial regions characterized by $Q>0$ and $Q<0$ are shown in black and white, respectively. 
Surfaces of $c(\mathbf{x}, t)=0.01$ and $c(\mathbf{x}, t)=0.5$ are shown in blue and red solid lines, respectively. Axial $x$ and transverse $y$ coordinates are reported in $\mathrm{mm}, z=2 \mathrm{~mm}$.

Since (i) the primary effect of a turbulent velocity field on a premixed flame consists in stretching the flame, (ii) the local rate of an increase in the flame surface area $A_{f}$ is controlled by the local stretch rate $\dot{s}$, i.e. $d \ln A_{f} / d t=\dot{s}$ [19,55-58], and (iii) the local stretch rate is equal to $\dot{s}=a_{t}+S_{d} \nabla \cdot \mathbf{n}$; it is of interest to compare direct contributions of the potential and rotational turbulent velocity fields to the stretch rate by comparing the strain rates $a_{t}=\nabla$. $\mathbf{u}-\mathbf{n n}: \nabla \mathbf{u}$ induced by the two fields. Here, $\mathbf{n}=-\nabla c /|\nabla c|$ is the unit vector normal to the local flame surface $c(\mathbf{x}, t)=c_{f}, S_{d}=[\nabla \cdot(\rho D \nabla c)+W] /(\rho|\nabla c|)$ is the local displacement speed [34,58], $D$ is the molecular diffusivity of $c$, and $W$ is the mass rate of product creation.

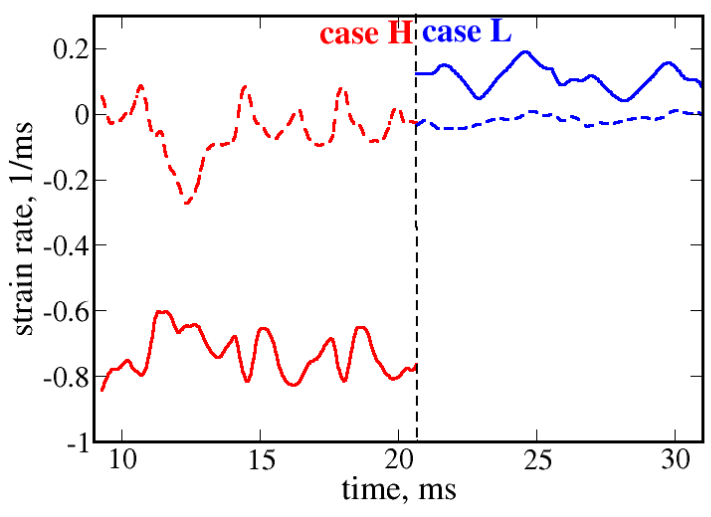

(a)

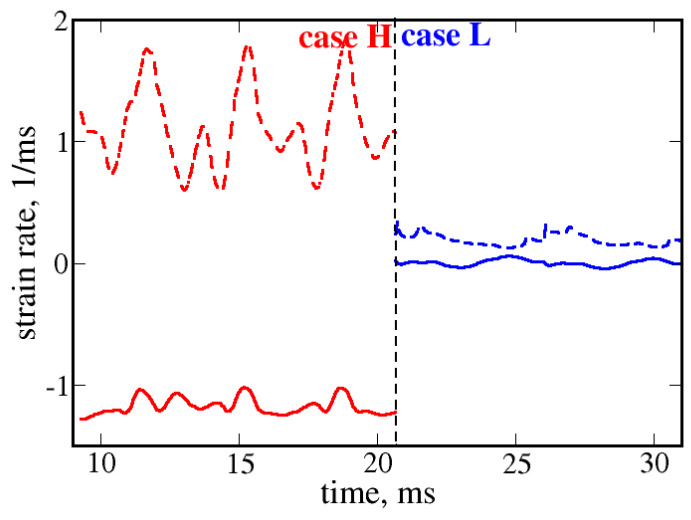

(b)

Figure 4. Strain rates conditioned to (a) cold flamelet edge, i.e. $\left\langle a_{t} \mid 0.001<c(\mathbf{x}, t)<0.03\right\rangle$, and (b) reaction-zone, i.e. $\left\langle a_{t} \mid 0.75<c(\mathbf{x}, t)<0.97\right\rangle$, and averaged over the entire volume of the flame brush vs. time. Solid and dashed lines show contributions from the rotational and potential velocity fields, respectively. Red and blue lines show results obtained in cases $\mathrm{H}$ and

$\mathrm{L}$, respectively.

Figure 4a shows that the volume-averaged contribution from the rotational velocity field to the strain rate conditioned to a cold flamelet edge is negative in case $\mathrm{H}$, but positive in case $\mathrm{L}$, whereas the contribution from the potential velocity field to $\left\langle a_{t} \mid 0.001<c(\mathbf{x}, t)<0.03\right\rangle$ oscillates around zero in both cases. In the reaction zone associated with $W(c)>$ $\max \{W(c)\} / 2$, i.e. with $0.75<c(\mathbf{x}, t)<0.97$, (i) magnitudes of contributions from both fields are much larger in case $\mathrm{H}$ than in case $\mathrm{L}$ due to stronger combustion-induced local flow perturbations in the former case and (ii) contributions from the rotational (potential) velocity fields are negative (positive, respectively). It is worth remembering that combustion-induced thermal expansion affects the velocity field in the unburned reactants only indirectly (due to combustion induced pressure perturbations), but directly affects the velocity field within the reaction zone, where the local density varies.

Since the leading points on the instantaneous flame surface can play a crucial role in the propagation of premixed turbulent flames, as discussed in details elsewhere [59-65], comparison of contributions from the rotational and potential velocity fields to the local strain rate at the leading points is also of fundamental interest. For this purpose, two sets of leading points were extracted from the DNS data (i) by finding a leading plane such that $c(\mathbf{x}, t) \geq c^{*}$ somewhere on the plane, but $c(\mathbf{x}, t)<c^{*}$ everywhere upstream of the plane, and (ii) by selecting a point $\mathbf{x}_{l p}(t)$ on the leading plane, characterized by the largest $c(\mathbf{x}, t)$. Two sets of 
the leading points will be considered using $c^{*}=0.03$ and 0.75 . They are associated with flamelet preheat and reaction zones, respectively.

Figure 5a shows that strain rates generated by the potential and rotational velocity fields have opposite signs in the leading points found for the preheat zone in case $\mathrm{H}$. The rotational strain rate is negative, thus, reducing the local flamelet surface area, whereas the potential strain rate is positive, thus, increasing the area. Both strain rates are predominantly negative in the leading points found for the reaction zone in the same case, with the magnitude of the rotational strain rate being larger, see Fig. 5 b. Such effects are weakly pronounced in case L.

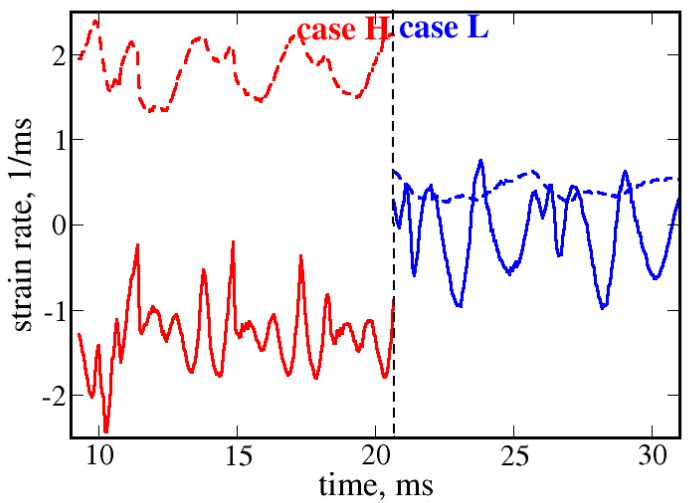

(a)

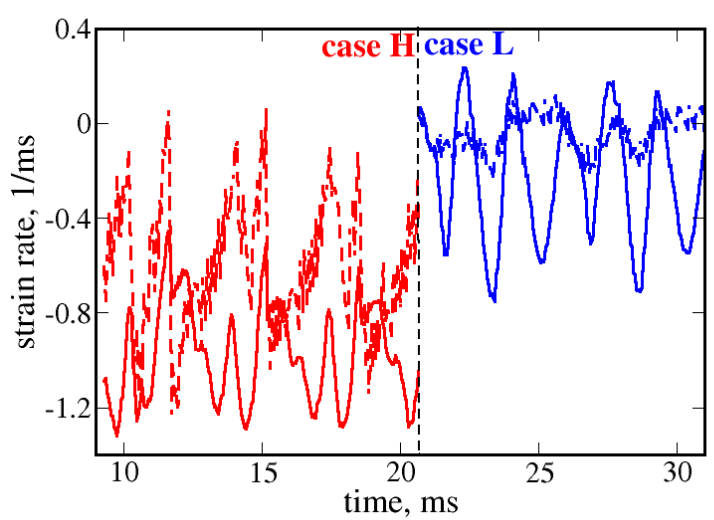

(b)

Figure 5. Strain rates $a_{t}$ in the leading points found for the (a) preheat and (b) reaction zones vs. time. Solid and dashed lines show contributions from the rotational and potential velocity fields, respectively. Red and blue lines show results obtained in cases $\mathrm{H}$ and $\mathrm{L}$, respectively.

\section{Conclusions}

The Helmholtz-Hodge decomposition was applied to velocity fields generated in an earlier DNS study $[14,15]$ of two weakly turbulent premixed flames associated with the flamelet combustion regime and characterized by significantly different density ratios. Comparison of the obtained rotational and potential velocity fields straightforwardly shows that combustioninduced thermal expansion can significantly change the local structure of the incoming constant-density turbulent flow of unburned reactants. In particular, stronger (weaker) potential (rotational, respectively) velocity fluctuations can be generated in the reactant flow within the flame brush. The effect magnitude is increased by the density ratio $\sigma$, with the effects being well (weakly) pronounced at $\sigma=7.53$ (2.50, respectively).

Moreover, in case $\mathrm{H}$ characterized by $\sigma=7.53$, generation of the potential velocity fluctuations is documented not only within the mean flame brush, but also upstream of it.

Furthermore, the potential and rotational velocity fields can cause opposite changes of the local area of an iso-scalar surface of $c(\mathbf{x}, t)=c_{f}$ within flamelets by generating the local strain rates of opposite signs. This phenomenon will further be explored in subsequent studies.

\section{Acknowledgements}

ANL gratefully acknowledges the financial support by the Chalmers Transport and Energy Areas of Advance, and by the Combustion Engine Research Center. VAS gratefully acknowledges the financial support by ONERA and by the Grant of the Ministry of Education and Science of the Russian Federation (Contract No. 14.G39.31.0001 of 13.02.2017). 


\section{References}

[1] Karlovitz, B., Denniston, D.W., Wells, F.E., "Investigation of turbulent flames", J. Chem. Phys. 19:541-547 (1951).

[2] Scurlock, A.C., Grover, J.H., "Propagation of turbulent flames", Proc. Combust. Inst. 4:645-658 (1953).

[3] Günther, R., "Turbulence properties of flames and their measurement", Prog. Energy Combust. Sci. 9:105-154 (1983).

[4] Bray, K.N.C., "Turbulent transport in flames", Proc. R. Soc. London A 451:231-256 (1995).

[5] Lipatnikov, A.N., Chomiak, J., "Effects of premixed flames on turbulence and turbulent scalar transport", Prog. Energy Combust. Sci. 36:1-102 (2010).

[6] Sabelnikov, V.A., Lipatnikov, A.N., "Recent advances in understanding of thermal expansion effects in premixed turbulent flames", Annu. Rev. Fluid Mech. 49:91-117 (2017).

[7] Swaminathan, N., Grout, R.W., "Interaction of turbulence and scalar fields in premixed flames", Phys. Fluids 18:045102 (2006).

[8] Chakraborty, N., Swaminathan, N., "Influence of the Damköhler number on turbulencescalar interaction in premixed flames. I. Physical insight", Phys. Fluids 19:045103 (2007).

[9] Steinberg, A.M., Driscoll, J.F., Swaminathan, N., "Statistics and dynamics of turbulence-flame alignment in premixed combustion", Combust. Flame 159:2576-2588 (2012).

[10] Sponfeldner, T., Boxx, I., Beyrau, F., Hardalupas, Y., Meier, W., Taylor, A.M.K.P., "On the alignment of fluid-dynamic principal strain-rates with the 3D flamelet-normal in a premixed turbulent V-flame", Proc. Combust. Inst. 35:1269-1276 (2015).

[11] Zhao, S., Er-Raiy, A., Bouali, Z., Mura, A., "Dynamics and kinematics of the reactive scalar gradient in weakly turbulent premixed flames", Combust. Flame 198:436-464 (2018).

[12] Lipatnikov, A.N., Chomiak, J., Sabelnikov, V.A., Nishiki, S., Hasegawa, T., "Unburned mixture fingers in premixed turbulent flames", Proc. Combust. Inst. 35:1401-1408 (2015).

[13] Lipatnikov, A.N., Chomiak, J., Sabelnikov, V.A., Nishiki, S., Hasegawa, T., “A DNS study of the physical mechanisms associated with density ratio influence on turbulent burning velocity in premixed flames", Combust. Theory Modell. 22:131-155 (2018).

[14] Nishiki, S., Hasegawa, T., Borghi, R., Himeno, R., "Modeling of flame-generated turbulence based on direct numerical simulation databases", Proc. Combust. Inst. 29:2017-2022 (2002).

[15] Nishiki, S., Hasegawa, T., Borghi, R., Himeno, R., "Modelling of turbulent scalar flux in turbulent premixed flames based on DNS databases", Combust. Theory Modell. 10:3955 (2006).

[16] Carter, C.D., Hammack, S., Lee, T., "High-speed flamefront imaging in premixed turbulent flames using planar laser-induced fluorescence of the $\mathrm{CH} \mathrm{C}-\mathrm{X}$ band", Combust. Flame 168:66-74 (2016).

[17] Chowdhury, B.R., Cetegen, B.M., "Experimental study of the effects of free stream turbulence on characteristics and flame structure of bluff-body stabilized conical lean premixed flames", Combust. Flame 178:301-328 (2017).

[18] Landau, L.D., Lifshitz, E.M., Fluid Mechanics, Pergamon Press, 1987.

[19] Clavin, P., "Dynamical behavior of premixed flame fronts in laminar and turbulent flows", Prog. Energy Combust. Sci. 11:1-59 (1985). 
[20] Matalon, M., "Intrinsic flame instabilities in premixed and nonpremixed combustion", Annu. Rev. Fluid Mech. 39:163-191 (2007).

[21] Sabelnikov, V.A., Lipatnikov, A.N., Nishiki, S., Hasegawa, T., "Application of conditioned structure functions to exploring influence of premixed combustion on twopoint turbulence statistics", Proc. Combust. Inst. 37, in press.

[22] Tsinober, A., An Informal Conceptual Introduction to Turbulence, Springer, 2009.

[23] Carlsson, H., Yu, R., Bai, X.-S., "Flame structure analysis for categorization of lean premixed $\mathrm{CH}_{4}$ /air and $\mathrm{H}_{2}$ /air flames at high Karlovitz numbers: Direct numerical simulation studies", Proc. Combust. Inst. 35:1425-1432 (2015).

[24] Uranakara, H.A., Chaudhuri, S., Dave, H.L., Arias, P.G., Im, H.G., "A flame particle tracking analysis of turbulence-chemistry interaction in hydrogen-air premixed flames", Combust. Flame 163:220-240 (2016).

[25] Aspden, A.J., Day, M.J., Bell, J.B., "Three-dimensional direct numerical simulation of turbulent lean premixed methane combustion with detailed kinetics", Combust. Flame 165:266-283 (2016).

[26] Cecere, D., Giacomazzi, E., Arcidiacono, N.M., Picchia, F.R., "Direct numerical simulation of a turbulent lean premixed $\mathrm{CH}_{4} / \mathrm{H}_{2}$-air slot flame", Combust. Flame 165:384-401 (2016).

[27] Bobbitt, B., Lapointe, S., Blanquart, G., "Vorticity transformation in high Karlovitz number premixed flames", Phys. Fluids 28:015101 (2016).

[28] Wang, H., Hawkes, E.R., Zhou, B., Chen, J.H., Li, Z., Aldén, M., "A comparison between direct numerical simulation and experiment of the turbulent burning velocityrelated statistics in a turbulent methane-air premixed jet flame at high Karlovitz number", Proc. Combust. Inst. 36:2045-2053 (2017).

[29] Chaudhuri, S., Kolla, H., Dave, H.L., Hawkes, E.R., Chen, J.H., Law, C.K., "Flame thickness and conditional scalar dissipation rate in a premixed temporal turbulent reacting jet", Combust. Flame 184:273-285 (2017).

[30] MacArt, J.F., Grenga, T., Mueller, M.E., "Effects of combustion heat release on velocity and scalar statistics in turbulent premixed jet flames at low and high Karlovitz numbers", Combust. Flame 191:468-485 (2018).

[31] Minamoto, Y., Yenerdag, B., Tanahashi, M., "Morphology and structure of hydrogen-air turbulent premixed flames", Combust. Flame 192:369-383 (2018).

[32] Borghi, R., "On the structure and morphology of turbulent premixed flames", Recent Advances in Aerospace Science, Eds. by Casci, S., Bruno, C., Plenum, 1984, pp. 117138.

[33] Williams, F.A., Combustion Theory, 2nd ed., Benjamin/Cummings, 1985, p. 412.

[34] Peters, N., Turbulent Combustion, Cambridge University Press, 2000.

[35] Lipatnikov, A.N., Nishiki, S., Hasegawa, T., "DNS assessment of relation between mean reaction and scalar dissipation rates in the flamelet regime of premixed turbulent combustion", Combust. Theory Modell. 19:309-328 (2015).

[36] Im, Y.H., Huh, K.Y., Nishiki, S., Hasegawa, T., "Zone conditional assessment of flamegenerated turbulence with DNS database of a turbulent premixed flame", Combust. Flame 137:478-488 (2004).

[37] Mura, A., Tsuboi, K., Hasegawa, T., "Modelling of the correlation between velocity and reactive scalar gradients in turbulent premixed flames based on DNS data", Combust. Theory Modell. 12:671-698 (2008).

[38] Mura, A., Robin, V., Champion, M., Hasegawa, T., "Small scale features of velocity and scalar fields in turbulent premixed flames", Flow Turbul. Combust. 82:339-358 (2009). 
[39] Robin, V., Mura, A., Champion, M., Hasegawa, T., "Direct and indirect thermal expansion effects in turbulent premixed flames", Combust. Sci. and Tech. 182:449-464 (2010).

[40] Robin, V., Mura, A., Champion, M., "Modeling of the effects of thermal expansion on scalar turbulent fluxes in turbulent premixed flames", J. Fluid Mech. 689:149-182 (2011).

[41] Bray, K.N.C., Champion, M., Libby, P.A., Swaminathan, N., "Scalar dissipation and mean reaction rates in premixed turbulent combustion", Combust. Flame 158:2017-2022 (2011).

[42] Lipatnikov, A.N., Nishiki, S., Hasegawa, T., "A direct numerical simulation study of vorticity transformation in weakly turbulent premixed flames", Phys. Fluids 26:105104 (2014).

[43] Lipatnikov, A.N., Sabelnikov, V.A., Nishiki, S., Hasegawa, T., Chakraborty, N., "DNS assessment of a simple model for evaluating velocity conditioned to unburned gas in premixed turbulent flames", Flow Turbul. Combust. 94:513-526 (2015).

[44] Sabelnikov, V.A., Lipatnikov, A.N., Chakraborty, N., Nishiki, S., Hasegawa, T., "A transport equation for reaction rate in turbulent flows", Phys. Fluids 28:081701 (2016).

[45] Sabelnikov, V.A., Lipatnikov, A.N., Chakraborty, N., Nishiki, S., Hasegawa, T., "A balance equation for the mean rate of product creation in premixed turbulent flames", Proc. Combust. Inst. 36:1893-1901 (2017).

[46] Lipatnikov, A.N., Sabelnikov, V.A., Nishiki, S., Hasegawa, T., "Flamelet perturbations and flame surface density transport in weakly turbulent premixed combustion", Combust. Theory Modell. 21:205-227 (2017).

[47] Lipatnikov, A.N., Sabelnikov, V.A., Chakraborty, N., Nishiki, S., Hasegawa, T., "A DNS study of closure relations for convection flux term in transport equation for mean reaction rate in turbulent flow", Flow Turbul. Combust. 100:75-92 (2018).

[48] Lipatnikov, A.N., Sabelnikov, V.A., Nishiki, S., Hasegawa, T., "Combustion-induced local shear layers within premixed flamelets in weakly turbulent flows", Phys. Fluids 30:085101 (2018).

[49] Lipatnikov, A.N., Sabelnikov, V.A., Nishiki, S., Hasegawa, T., "Does flame-generated vorticity increase turbulent burning velocity?" Phys. Fluids 30:081702 (2018).

[50] Lipatnikov, A.N., Nishiki, S., Hasegawa, T., "A DNS assessment of linear relations between filtered reaction rate, flame surface density, and scalar dissipation rate in a weakly turbulent premixed flame", Combust. Theory Modell. 23, in press.

[51] Kraichnan, R.H., "Decay of isotropic turbulence in the direct-interaction approximation", Phys. Fluids 7:1030-1048 (1964).

[52] Abraham, R., Marsden, J.E., Ratiu, T., Tensor Analysis and Applications, 2nd ed., Springer, 1988.

[53] Chorin, A.J., Marsden, J.E., A Mathematical Introduction to Fluid Mechanics, Springer, 1993.

[54] Denaro, F.M., "On the application of Helmholtz-Hodge decomposition in projection methods for incompressible flows with general boundary conditions", Int. J. Numerical Methods in Fluids 43:43-69 (2003).

[55] Matalon, M., "On flame stretch", Combust. Sci. and Tech. 31:169-182 (1983).

[56] Pope, S.B., "The evolution of surface in turbulence", Int. J. Engng Sci. 26:445-469 (1988).

[57] Candel, S., Poinsot, T., "Flame stretch and the balance equation for the flame area", Combust. Sci. and Tech. 170:1-15 (1990).

[58] Veynante D., Vervisch, L., "Turbulent combustion modeling", Prog. Energy Combust. Sci. 28:193-266 (2002). 
[59] Kuznetsov, V.R., Sabelnikov, V.A., Turbulence and Combustion, Hemisphere, 1990.

[60] Lipatnikov, A.N., Chomiak, J., "Molecular transport effects on turbulent flame propagation and structure", Prog. Energy Combust. Sci. 31:1-73 (2005).

[61] Venkateswaran, P., Marshall, A., Seitzman, J., Lieuwen, T., "Scaling turbulent flame speeds of negative Markstein length fuel blends using leading points concepts", Combust. Flame 162:375-387 (2015).

[62] Sabelnikov, V.A., Lipatnikov, A.N., "Transition from pulled to pushed fronts in premixed turbulent combustion: theoretical and numerical study", Combust. Flame 162:2893-2903 (2015).

[63] Kim, S.H., "Leading points and heat release effects in turbulent premixed flames", Proc. Combust. Inst. 36:2017-2024 (2017).

[64] Dave, H.L., Mohan, A., Chaudhuri, S., "Genesis and evolution of premixed flames in turbulence", Combust. Flame 196:386-399 (2018).

[65] Lipatnikov, A.N., Chakraborty, N., Sabelnikov, V.A., "Transport equations for reaction rate in laminar and turbulent premixed flames characterized by non-unity Lewis number", Int. J. Hydrogen Energy 43:21060-21069 (2018). 